\title{
Saturated absorption spectroscopy: elimination of crossover resonances by use of a nanocell
}

\author{
A. Sargsyan, D. Sarkisyan, A. Papoyan \\ Institute for Physical Research, National Academy of Sciences of Armenia, Ashtarak, 0203 Armenia \\ Y. Pashayan-Leroy \\ Laboratoire de Physique de l'Université de Bourgogne, UMR-CNRS 5027, 21078 Dijon Cedex, France
}

P. Moroshkin, A. Weis

Départment de Physique, Université de Fribourg,

Chemin du Musée 3, 1700 Fribourg, Switzerland

A. Khanbekyan, E. Mariotti, and L. Moi

Department of Physics, University of Siena, Via Roma 56, 53100 Siena, Italy

\begin{abstract}
It is demonstrated that velocity selective optical pumping/saturation resonances of reduced absorption in a $\mathrm{Rb}$ vapor nanocell with thickness $L=\lambda, 2 \lambda$, and $3 \lambda$ (resonant wavelength $\lambda=780$ $\mathrm{nm}$ ) allow the complete elimination of crossover $(\mathrm{CO})$ resonances. We observe well pronounced resonances corresponding to the $\mathrm{F}_{g}=3 \rightarrow \mathrm{F}_{e}=2,3,4$ hyperfine transitions of the ${ }^{85} \mathrm{Rb}_{2}$ line with linewidths close to the natural width. A small $\mathrm{CO}$ resonance located midway between $\mathrm{F}_{g}=3 \rightarrow$ $\mathrm{F}_{e}=3$ and $\mathrm{F}_{g}=3 \rightarrow \mathrm{F}_{e}=4$ transitions appears only for $L=4 \lambda$. The $\mathrm{D}_{2}$ line $(\lambda=852 \mathrm{~nm})$ in a Cs nanocell exhibits a similar behavior. From the amplitude ratio of the CO and VSOP resonances it is possible to determine the thickness of the column of alkali vapor in the range of $1-1000 \mu \mathrm{m}$. The absence of $\mathrm{CO}$ resonances for nanocells with $L \sim \lambda$ is attractive for frequency reference application and for studying transitions between Zeeman sublevels in external magnetic fields.
\end{abstract}

PACS numbers: 32.70.Jz; 42.62.Fi; 32.10.Fn; 42.50.Hz

Key words: laser spectroscopy; atomic spectra; saturated absorption; thin vapor cells; sub-Doppler spectroscopy.

\section{INTRODUCTION}

Saturated absorption (SA) spectroscopy is widely used in the realization of frequency references for atomic transitions [1-3]. In this technique the laser beam is split into a weak probe field and a strong pump field, which are sent to the interaction cell as counter-propagating overlapping beams. Because of opposite Doppler shifts, only the atoms moving perpendicular to the radiation propaga- tion direction resonantly interact with both laser beams. For these atoms, the pump beam saturates the transition, and the absorption spectrum of the probe shows a Doppler-free dip, the so called velocity selective optical pumping/saturation (VSOP) resonance located at the line center. With properly chosen pump and probe beam intensities, careful adjustment of the geometry, and elimination of stray magnetic fields, the linewidth of the resonance (to which we refer to as "VSOP" resonance) may be as narrow as the natural width of the transition.

The situation is more complex when two or more atomic (hyperfine) transitions overlap within the Doppler profile, which is the case for the majority of real atomic lines. The presence of multiple unresolved hyperfine transitions results in the formation of so called crossover $(\mathrm{CO})$ resonances. These spurious resonances appear when the 
laser frequency is tuned midway between two transitions, so that for group of atoms moving with a certain longitudinal velocity the pump beam drives one transition, while the probe drives the other one linked to the former by the same ground state. As a result of the partial depletion of the ground state by the pump beam, the probe "sees" a reduced absorption. Thus, the VSOP process is also responsible for the occurrence of $\mathrm{CO}$ resonances. The CO resonances may seriously complicate the applicability of the SA spectral reference technique, notably in high-resolution spectroscopy of hyperfine levels with a small $(10 \div 30 \mathrm{MHz})$ frequency spacing. In that case the $\mathrm{CO}$ resonances mask the real atomic VSOP resonances and thus hinder of even impede the identification of the relevant spectral features. We note that, as a rule, the amplitude of the $\mathrm{CO}$ resonances are larger than the one of the actual VSOP resonances.

There are several techniques that allow the elimination of crossover resonances in atomic vapors. In [4] two co-propagating laser beams (a pump laser with fixed frequency and a probe laser with tunable frequency) were used rather than two counter-propagating beams. In [5] it was shown that the use of a thin (20 $\mu$ m-long) cell in conventional SA setup allows a reduction of the amplitude of $\mathrm{CO}$ resonance. Note, that the optical selective reflection (SR) spectroscopy technique [6,7] also allows one to eliminate CO resonances, but for a correct determination of an atomic transition position by this technique the spectra must undergo further non-trivial processing.

The aim of this work is to show that for very small (wavelength-scale) thicknesses $L$ of the atomic vapor column it is possible to completely eliminate the CO resonances, both in single-beam transmission spectroscopy and in the SA configuration.

\section{EXPERIMENTAL SETUP}

The scheme of the experimental setup is presented in Fig.1. The beam from a single-frequency tunable diode laser (1 mm diameter, power $P_{L}=30 \mathrm{~mW}$, central wavelength $\lambda=780 \mathrm{~nm}$, linewidth $\gamma_{L} \sim 5 \mathrm{MHz}$ ) irradiated a thin cell 1 under an incidence angle close to the normal. A Faraday isolator $(F I)$ was inserted to prevent optical

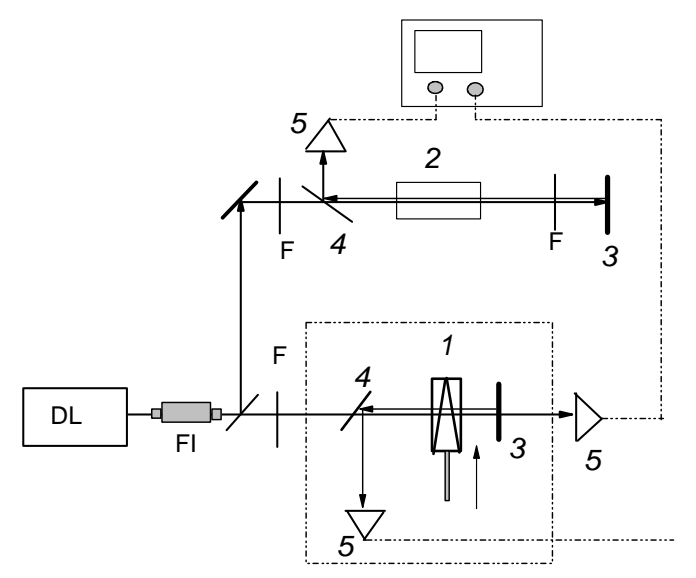

FIG. 1: Experimental setup. $D L$ - tunable diode laser, $\lambda=$ $780 \mathrm{~nm}(852 \mathrm{~nm})$; FI- Faraday isolator; 1 - Rb (Cs) cell with nanometric, micrometric or ordinary thickness (see the text); F- neutral density filters; 2- Rb (Cs) cell of ordinary length; 3- mirror; 4- glass plate; 5- photodiodes.

feedback to the laser. In the experiment we have used different cells 1 with various thicknesses $L$ in the ranges: $L=\lambda \div 6 \lambda$ (nanometric, vertical wedged-shaped vapor column) [8-13]; $9 \mu \mathrm{m}, 60 \mu \mathrm{m}, 700 \mu \mathrm{m}$ [14]; and $2 \mathrm{~mm}$, $4 \mathrm{~mm}, 60 \mathrm{~mm}$-long. The cell was inserted into an oven with two apertures for passing the radiation. The $\mathrm{Rb}$ density determining temperature of the cell's side-arm was adjusted in the range of $40 \div 115^{\circ} \mathrm{C}$, depending on the cell thickness. To prevent atomic vapour condensation on the cell windows their temperature was kept 20 to 30 degrees higher that the sidearm tempearture. The laser intensity was controlled by neutral density filters $F$ and its frequency scanned over $\sim 10 \mathrm{GHz}$, covering the hyperfine spectral components of the the ${ }^{85} \mathrm{Rb}_{2}$ lines $\mathrm{F}_{g}=3 \rightarrow \mathrm{F}_{e}=2,3,4$, where the subscripts $g$, $e$ refer to the lower and upper levels, respectively). A mirror 3 and a glass plate 4 (which are shown in Fig.1 inside the dotted rectangle) were used to form the SA spectra. In order to vary thicknesses $\mathrm{L}$ of the vapour column in 
the nanocell in the range from $L=(1-6) \lambda$ the oven was translated along the vertical direction as indicated by the arrow in Fig.1. A part of the laser beam was sent to a normal vapor cell with a thickness of $60 \mathrm{~mm}$ to obtain a reference SA spectrum. The upper set of filters $F$ was used to properly attenuate the laser beam in order to get the reference SA spectra with a linewidth close to the natural width. The spectra were registered in a four-channel digital storage oscilloscope (Tektronix, TDS 2014B).

\section{RESULTS AND DISCUSSION}

It was shown earlier [9-13] that the ratio $L / \lambda$, where $L$ is the thickness of the atomic vapor column and $\lambda$ the laser wavelength resonant with the atomic transition, is an important parameter that determines the widths, shapes and amplitudes of the absorption resonances in a nanocell. In particular, it was shown that the spectral width of resonant absorption is minimal for $L=(2 n+1)$ $\lambda / 2$ (where $n$ is an integer), an effect which was called "Dicke-type Coherent Narrowing Effect" (DCNE). It was also shown that for $L=n \lambda$ the spectral width of the resonant absorption reaches a maximal value close to the Doppler width (about several hundreds of $\mathrm{MHz}$ ), an effect called collapse of DCNE $[9,10]$. In [11] the DCNE effect and the collapse of DCNE were investigated up to the thicknesses $L=7 \lambda / 2$ for the $\mathrm{D}_{2}$ line of ${ }^{85} \mathrm{Rb}$ at $\lambda=$ $780 \mathrm{~nm}$. Here we consider the case where $L=n \lambda$, since as it was shown in $[10,11]$ that the VSOP resonances appear under this condition. In Fig.2 the transmission spectra are presented for $L$ varying in the range from $L=\lambda$ up to $6 \lambda$ with steps of $\lambda$ (for these recordings the mirror 3 and the glass plate 4 were removed). The effective laser intensity (EI) is determined by multiplying the measured intensity by the coefficient $\left[\gamma_{N} /\left(\gamma_{N}+\gamma_{L}\right]\right.$, where $\gamma_{N}$ is the spontaneous decay rate of the $\mathrm{Rb}_{2}$ line, $\gamma_{L} \sim 5 \mathrm{MHz}$, and $\mathrm{EI}$ is $10 \mathrm{~mW} / \mathrm{cm}^{2}$. When $L$ is varied from $L=\lambda$ to $3 \lambda$ it can be seen that only the VSOP resonances are detected. These peaks of decreased absorption are located

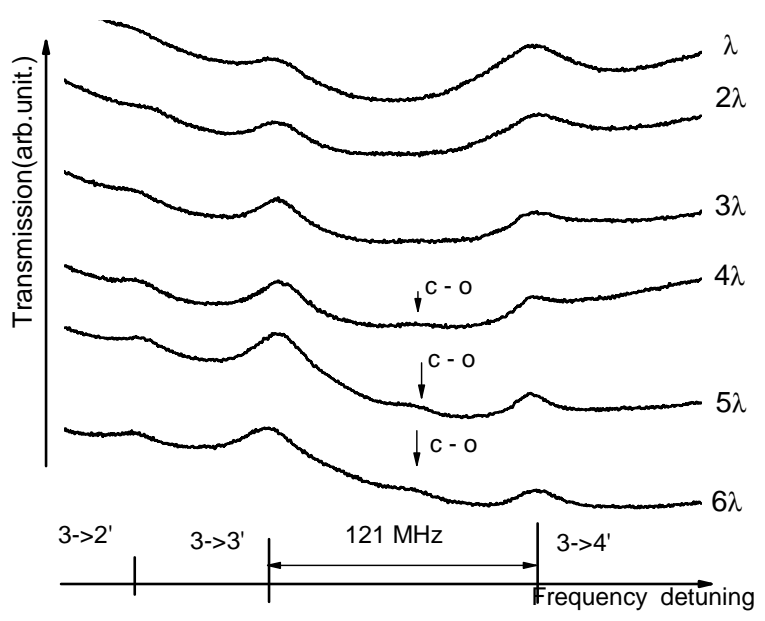

FIG. 2: Transmission spectra of the nanocell for the transitions $\mathrm{F}_{g}=3 \rightarrow F_{e}=2,3,4$ of ${ }^{85} \mathrm{Rb}_{2}$ line, for $L$ varying in the range $\lambda \div 6 \lambda$. The effective laser intensity was $\sim 10$ $\mathrm{mW} / \mathrm{cm}^{2}$. The temperature of the cell's side-arm is $115^{\circ} \mathrm{C}$. The CO resonances are marked by arrows.

exactly at the the atomic transition frequencies [5,10-14], and arise because the atom in the ground level $\mathrm{F}_{g}=3$ absorbs a laser photon populating the excited level, followed by spontaneous decay to the ground level $\mathrm{F}_{g}=2$ or $\mathrm{F}_{g}=3$, an effect well known as optical pumping (OP) $[1-3,5,15]$ (peculiarities of OP process in sub-millimeter thin cell are presented in $[16,17])$. As a result, a fraction of the atoms populates the $\mathrm{F}_{g}=2$ level, and the number of atoms absorbing from the $\mathrm{F}_{g}=3$ level is reduced. As a consequence absorption from this level decreases. The efficiency of OP is determined by the expression

$$
\eta \sim \frac{\Omega^{2} \gamma_{N} t}{(\Delta+\vec{k} \vec{v})^{2}+\Gamma^{2}}
$$

where $t$ is the average interaction time of the atom with the radiation field, $v$ the atomic velocity, $\Delta$ the detuning, $\Gamma$ the sum of homogeneous and inhomogeneous broadenings, and $k=2 \pi / \lambda$ [15]. Eq. (11) shows how the optical pumping efficiency grows with the interaction time $t$. For atoms flying perpendicularly to the laser beam the interaction time is $t_{D}=D / v$, where $D$ is the 
laser beam diameter, while atoms flying along the laser beam have an interaction time of $t_{L}=L / v$. Since D $1 \mathrm{~mm}$, and $L=780 \mathrm{~nm}$ (or $852 \mathrm{~nm}$ in the case of Cs), $t_{D}$ exceeds $t_{L}$ by three orders of magnitude. For atoms flying perpendicular to the laser beam $\mathbf{k v}=0$, and the efficiency in Eq. (1) becomes maximal for $\Delta=0$. For this reason the VSOP peak is centered exactly at the atomic transition frequency $[5,10-14]$. Note, that there is

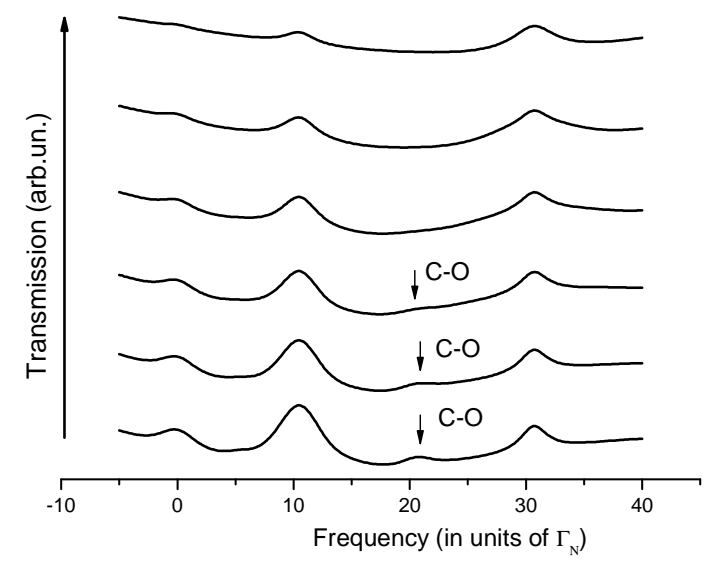

FIG. 3: Calculated spectra for the conditions of Fig.2. Rabi frequency $\Omega=0.4 \gamma_{N} ; \gamma_{L}=5 \mathrm{MHz} ; \gamma_{N}=6 \mathrm{MHz}$.

a reflected beam from the inner surface of the nanocell which propagates backwards with respect to the main beam, and this beam may cause the formation of $\mathrm{CO}$ resonance. However, the interaction time $t_{L}$ for atoms with a longitudinal velocity $v_{z}=2 \pi \varepsilon / k$ (where $\varepsilon$ is half of the frequency separation of corresponding excited levels) along the laser beam is rather small for $L=\lambda, 2 \lambda$, and $3 \lambda$ to provide an efficient optical pumping which is needed for CO resonansce formation (in our case $2 \varepsilon$ is $121 \mathrm{MHz}$ and $63 \mathrm{MHz}$, respectively).

However, as can be seen from Fig.2, a small CO resonance (marked by arrows) located midway between the $\mathrm{F}_{g}=3 \rightarrow F_{e}=3$ and $\mathrm{F}_{g}=3 \rightarrow F_{e}=4$ transitions appears in the transmission spectra when the thickness $L=$ $4 \lambda$. One can also see a different behavior of VSOP peaks at different transitions. It is interesting to note that when
$L=\lambda$ the amplitude ratios of the VSOP peaks is close to the ratio of the atomic probabilities of the corresponding transitions $\mathrm{F}_{g}=3 \rightarrow F_{e}=2,3$, 4 , i.e. $A\left(3-4^{\prime}\right) / A\left(3-3^{\prime}\right) \sim$ 2, $A\left(3-3^{\prime}\right) / A\left(3-2^{\prime}\right) \sim 3[18]$. As the thickness increases, the amplitude of the VSOP peak corresponding to the $\mathrm{F}_{g}=3 \rightarrow F_{e}=4$ cycling transition decreases, while the amplitudes of the VSOP peaks for the non-cycling transitions $\mathrm{F}_{g}=3 \rightarrow F_{e}=2,3$ increase. In particular, for $L=$ $6 \lambda$ the ratio $A\left(3-4^{\prime}\right) / A\left(3-3^{\prime}\right) \sim 0.5$. Conversely, the ratio of the non-cycling transitions is practically independent of the thickness, i.e. $A\left(3-3^{\prime}\right) / A\left(3-2^{\prime}\right) \sim 3$. Fig. 3 presents the theoretical spectra for the same group of transitions, with the Rabi frequency $\Omega=0.4 \gamma_{N}, \gamma_{L}=5 \mathrm{MHz}$, and $\gamma_{N}=6 \mathrm{MHz}$. One sees that the theoretical model presented in [19] correctly describes the peculiarities of the VSOP of the cycling and non-cycling transitions as well as the behavior of the $\mathrm{CO}$ resonance as a function of the thickness $L$. One can conclude from Figs. 2 and 3 that the optimum condition for the formation of an atomic reference spectrum (e.g., to determine the frequency position of a weak atomic transition as $\mathrm{F}_{g}=3 \rightarrow F_{e}=2$ ) is obtained for $L=3 \lambda$, in which case narrow and large VSOP peaks are observed, along with the absence of CO resonances.

The second set of measurements was done for the same group of transitions employing SA configuration (mirror 3 and glass plate 4 are mounted as shown in Fig.1). Thin cells with thicknesses $L=\lambda(780 \mathrm{~nm}), 5 \lambda(3.9 \mu \mathrm{m}), 9 \mu \mathrm{m}$, $60 \mu \mathrm{m}, 700 \mu \mathrm{m}$ and cells with an length of $2 \mathrm{~mm}, 4 \mathrm{~mm}$ and $60 \mathrm{~mm}$ have been used. In Fig. 4 the SA spectra are presented for $L=\lambda(780 \mathrm{~nm}), 5 \lambda(3.9 \mu \mathrm{m}), 9 \mu \mathrm{m}$. The lower curve is the SA spectrum recorded in a $60 \mathrm{~mm}$ long cell serving as a reference. As before the $\mathrm{CO}$ resonance is completely absent in the case of $L=\lambda$, while it appears when $L=5 \lambda(3.9 \mu \mathrm{m})$ and the amplitude of the CO resonance increases as $L$ increases from $3.9 \mu \mathrm{m}$ to $9 \mu \mathrm{m}$. Fig. 5 shows the SA spectra for $L=60 \mu \mathrm{m}, 0.7 \mathrm{~mm}, 2$ $\mathrm{mm}$, and $4 \mathrm{~mm}$. The VSOP resonance of the $\mathrm{F}_{g}=3 \rightarrow$ $\mathrm{F}_{e}=4$ transition is sensitive to the polarizations of the pump and probe beams [20]. For a quantitative description it is therefore more convenient to use the ratio of the amplitude of the $\mathrm{CO}$ resonance appearing when the 


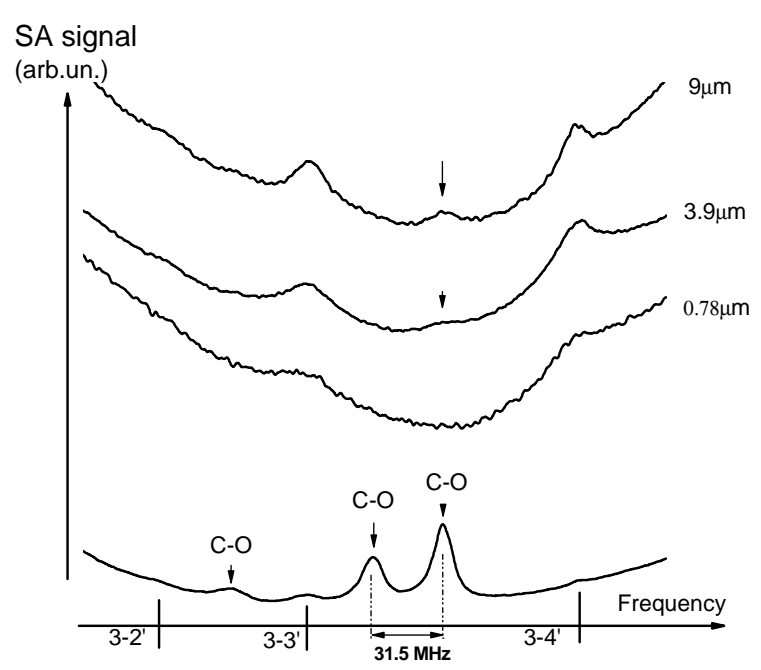

FIG. 4: Saturated absorption spectra for Rb cells with $L=$ $780 \mathrm{~nm}, 3.9 \mu \mathrm{m}, 9 \mu \mathrm{m}$. The lower curve is the SA spectrum of an ordinary $60 \mathrm{~mm}$ long cell. The CO resonances are marked by arrows.

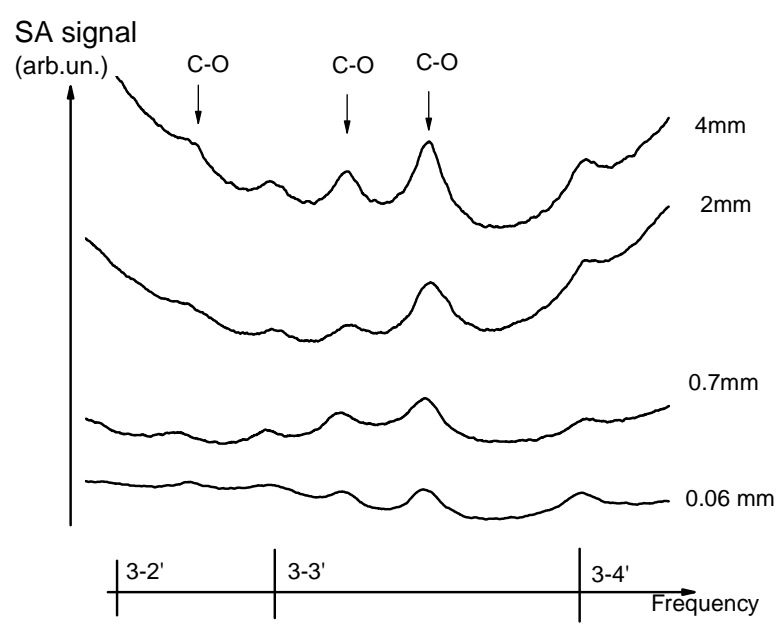

FIG. 5: Saturated absorption spectra for Rb cells with $L=$ $60 \mu \mathrm{m}, 0.7 \mathrm{~mm}, 2 \mathrm{~mm}$, and $4 \mathrm{~mm}$.

laser is tuned midway between $\mathrm{F}_{g}=3 \rightarrow F_{e}=3$ and $\mathrm{F}_{g}=3 \rightarrow F_{e}=4$ atomic transitions (this is the largest $\mathrm{CO}$ resonance in the spectrum), and the amplitude of the VSOP resonance of the $\mathrm{F}_{g}=3 \rightarrow F_{e}=3$ transition. Fig.6 shows the ratio of $A(\mathrm{CO}) / A\left(\mathrm{VSOP} 3-3^{\prime}\right)$ as a function of the cell thickness. The monotonic smooth increase of this dependence permits to infer the cell thickness from a measurement of the amplitude ratio. Note, that in the case of thinner cells $(L \rightarrow \lambda)$ the interferometric method presented in [9] allows the determination of the thickness $L$ with high accuracy $\sim 15 \mathrm{~nm}$ ). It should be noted that the dependence shown in Fig.6 is slightly affected by the pump and probe beam intensities, and is correct for the ${ }^{85} \mathrm{Rb}, \mathrm{F}_{g}=3 \rightarrow F_{e}=2,3,4$ atomic transitions.

There are several reasons which influence the $\mathrm{CO}$ resonance amplitude, in particular the frequency separation between the upper levels $(2 \varepsilon)$, the probabilities of the atomic transitions involved in the $\mathrm{CO}$ resonance formation, the thermal atomic velocity of the alkali atom $[2,3]$. A frequency-tunable distributed feedback diode laser with $\lambda=852 \mathrm{~nm}$ (linewidth $5 \mathrm{MHz}$ ) and $\mathrm{EI} \sim 10$ $\mathrm{mW} / \mathrm{cm}^{2}$ has been used to obtain the transmission spectra (see Fig.7) for a nanocell filled with Cs vapor for $\mathrm{L}$ varying in the range of $\lambda$ to $6 \lambda$ in steps of $\lambda\left(\mathrm{F}_{g}=4 \rightarrow\right.$ $F_{e}=3,4,5$ transitions of $\mathrm{D}_{2}$ line). In this case a small CO resonance appears at $L=6 \lambda$ when the laser is tuned midway between the $\mathrm{F}_{g}=4 \rightarrow F_{e}=4$ and $\mathrm{F}_{g}=4 \rightarrow$ $F_{e}=5$ atomic transitions. This is in agreement with the results presented in [5], where the ratio $A(\mathrm{CO}) / A$ (VSOP $\left.4-4^{\prime}\right)$ is $\sim 0.4$ for the $\mathrm{F}_{g}=4 \rightarrow F_{e}=3,4,5$ transitions in Cs and $L=100 \mu \mathrm{m}$, while for the $\mathrm{F}_{g}=3 \rightarrow F_{e}=2,3,4$ group in ${ }^{85} \mathrm{Rb}$ the ratio $A(\mathrm{CO}) / A\left(\operatorname{VSOP} 3-3^{\prime}\right)$ is $\sim 2$ at an even smaller cell thickness $(L=60 \mu \mathrm{m})$ (see Fig.5).

The difference of the SA spectra for different atomic transitions is well seen if we compare spectra obtained in similar conditions (i.e., cell length, temperature, laser intensity, etc.), as is the case for the lower traces of Figs. $4,7\left({ }^{85} \mathrm{Rb} \mathrm{D}_{2}\right.$ line, $\mathrm{F}_{g}=3 \rightarrow F_{e}=2,3,4$ in Fig.4, and Cs $\mathrm{D}_{2}$ line, $\mathrm{F}_{g}=4 \rightarrow F_{e}=3,4,5$ in Fig.7). For ${ }^{85} \mathrm{Rb}$ the ratio $A(\mathrm{CO}) / A\left(\mathrm{VSOP} 3-3^{\prime}\right)$ is $\sim 10$, while for $\mathrm{Cs}$ we find $A(\mathrm{CO}) / A\left(\mathrm{VSOP} 4-4^{\prime}\right) \sim 2$. Similar ratios can be also seen in $[2,3]$. From this behavior one can expect that for the case of the $\mathrm{Cs}_{g}=4 \rightarrow F_{e}=3,4,5$ transitions the increase of the $\mathrm{CO}$ resonance amplitude with the thickness should be somewhat weaker. It will be interesting to study the dependence of amplitudes ratio $A(\mathrm{CO}) / A(\mathrm{VSOP})$ on the atomic $\mathrm{D}_{1}$ line of $\mathrm{Na}$, since the value of $2 \varepsilon$ in this case is $190 \mathrm{MHz}$, i.e., is close to that of ${ }^{85} \mathrm{Rb}$, while the atomic velocity for $\mathrm{Na}$ is approximately 

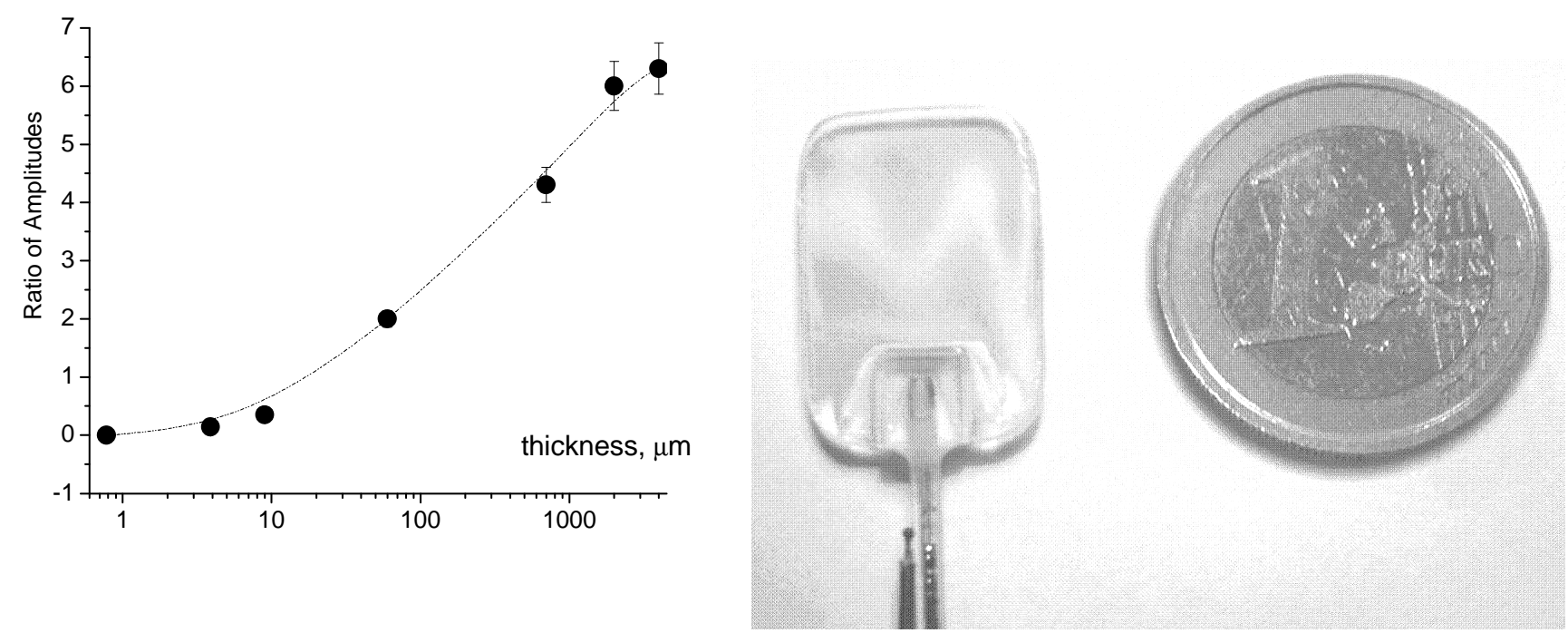

FIG. 6: Amplitude ratios $A(\mathrm{CO}) / A\left(\mathrm{VSOP} 3-3^{\prime}\right)$ as a function of the cell thickness. The dotted line is shown to guide the eye.

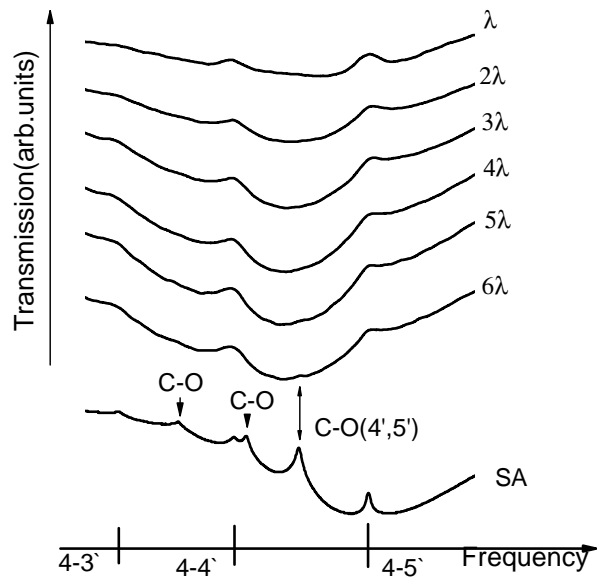

FIG. 8: Photograph of a nanocell filled with Na. A 1 Euro coin is shown for the scaling.

three times larger. For this purpose we have recently prepared a nanocell filled with $\mathrm{Na}$ metal (Fig.8). The thickness $L$ of the gap between the windows has a wedge in the vertical direction, varying from $100 \mathrm{~nm}$ to $550 \mathrm{~nm}$ at room temperature. At the windows' operating temperature of $\sim 200{ }^{\circ} \mathrm{C}$ the maximum thickness $L$ slightly increases, reaching $800 \mathrm{~nm}$. Thus, there are two important regions of the thickness $L=\lambda / 2$ and $L=\lambda$ (for $\mathrm{Na}$, $\lambda=590 \mathrm{~nm}$ ), where sub-Doppler absorption and fluorescence spectroscopy can be realized. This experiment is in progress at Siena University.

\section{CONCLUSION}

We have demonstrated that the use of a nanocell with a thickness $L=\lambda, 2 \lambda$ and $3 \lambda$ allows one to completely eliminate the CO resonance, while VSOP resonances lo-

FIG. 7: Transmission spectra of the Cs nanocell for the transitions $\mathrm{F}_{g}=4 \rightarrow F_{e}=3,4,5$ of ${ }^{133} \mathrm{Cs}_{2}$ line, for $L$ varying in the range $\lambda \div 6 \lambda$. The effective laser intensity was $\sim 10$ $\mathrm{mW} / \mathrm{cm}^{2}$. The temperature of the side-arm was $110{ }^{\circ} \mathrm{C}$. The lower curve is the SA spectrum in an ordinary cell. The CO resonances are marked by arrows.

cated on atomic transitions are well pronounced. The advantage of the use of nanocell with thickness $L=\lambda$ as a frequency reference for an atomic transition is that the ratio of the amplitudes for the VSOP peaks is close to the ratio of the atomic probabilities of the corresponding transitions. If, however, the center frequency of a weak atomic transition has to be determined, the use of 
a nanocell with a thickness $L=3 \lambda$ is more appropriate. If the thickness $L=4 \lambda$ for ${ }^{85} \mathrm{Rb}\left(L=6 \lambda\right.$ for ${ }^{133} \mathrm{Cs}$ ), a small CO resonance appears in the transmission spectra of ${ }^{85} \mathrm{Rb}$, located midway between the $\mathrm{F}_{g}=3 \rightarrow F_{e}=3$ and $\mathrm{F}_{g}=3 \rightarrow F_{e}=4$ transitions $\left(\mathrm{F}_{g}=4 \rightarrow F_{e}=4\right.$ and $\mathrm{F}_{g}=4 \rightarrow F_{e}=5$ in the case of $\mathrm{Cs}$ ).

For spectral reference applications the use of a singlebeam transmission spectrum of a nanocell has obvious advantages compared to the SA geometry, which requires counter-propagating beams in the nanocell.

From the ratio of the amplitudes of the CO and VSOP resonances, it is possible to determine the thickness of the alkali vapor column in the range from 1 to $1000 \mu \mathrm{m}$.

It is important to note that the absence of CO resonances in a nanocell with $L \sim \lambda$ allows one to study the behavior of atomic transitions between the Zeeman sublevels in external magnetic field [21], since the short time of flight ensures that the $\mathrm{CO}$ resonances are absent, even when the VSOP resonance is split into several Zeeman components. Conversely, even if CO resonances are absent in SA spectrum of an ordinary cell (this happens when the frequency distance between upper levels is larger than the Doppler width of the atomic transition), the CO resonance may appear again (due to a large time of flight) when the VSOP resonance is split into a several components.

\section{ACKNOWLEDGMENTS}

D.S. is grateful to University of Siena for the hospitality. The authors A.S, D.S, A.P, Y.P-L, E.M. and L.M. are thankful for the financial support provided by INTAS South-Caucasus Grant 06-1000017-9001. A.S, D.S, A.P, P.M. and A.W. are thankful for financial support provided by SCOPES Grant IB7320-110684/1.

\section{REFERENCES}

1. W. Demtrder, Laser Spectroscopy (SpringerVerlag, Berlin, 1982).
2. O. Schmidt, K.-M. Knaak, R. Wynands, and D. Meschede, Appl. Phys. B. 59,167 (1994)

3. D.A. Smith and I.G. Hughes, Am. J. Phys. 72, 631 (2004).

4. A. Banerjee and V. Natarajan, Opt. Lett. 28, 1912 (2003).

5. S. Briaudeau, D. Bloch, and M. Ducloy, Phys. Rev. A 59, 3723 (1999).

6. R. Müller and A. Weis, Appl. Phys. B 66 (3), 323 (1998).

7. H. Failache,S. Saltiel, M. Fichet, D.Bloch, and M.Ducloy, Phys. Rev. Lett. 83, 5467 (1999).

8. D. Sarkisyan, D. Bloch, A. Papoyan, and M. Ducloy, Opt. Commun. 200, 201 (2001).

9. G. Dutier, A. Yarovitski et al., Europhys. Lett. 63 (1), 35 (2003).

10. D. Sarkisyan, T. Varzhapetyan et al., Phys. Rev. A 69, 065802 (2004).

11. D. Sarkisyan, T. Varzhapetyan, A. Papoyan, D. Bloch, and M. Ducloy, Proc. SPIE 6257, 625701 (2006).

12. A. Sargsyan, D. Sarkisyan, and A. Papoyan, Phys. Rev. A 73, 033803 (2006).

13. C. Andreeva, S. Cartaleva et al., Phys. Rev. A 76, 013837 (2007).

14. D. Sarkisyan, A. Sargsyan, A. Papoyan, and Y. Pashayan-Leroy, Proc. SPIE 6604, 660405 (2007).

15. G. Nikogosyan, D. Sarkisyan, and Yu. Malakyan, J. Opt. Technol. 71, 602 (2004).

16. A.Ch. Izmailov, K. Fukuda, M. Kinoshita, M. Tachikawa, Laser Phys. 14(1), 30 (2004).

17. A.Ch. Izmailov, Laser Phys. Lett. $\quad 3(3), 132$ (2006). 
18. D. Sarkisyan, T. Becker, A. Papoyan, P. Thoumany, and H. Walther, Appl. Phys. B 76, 625 (2003).

19. A. Sargsyan, D. Sarkisyan, Y. PashayanLeroy, C. Leroy, P. Moroshkin, and A. Weis, http://eprintweb.org/S/archive/physics/0707.0379.
20. C.P. Pearman, C.S. Adams, et al., J. Phys. B: At. Mol. Opt. Phys. 35, 5141 (2002).

21. T. Varzhapetyan, H. Hakhumyan, D. Sarkisyan, V. Babushkin, A. Atvars, and M. Auzinsh, J. Contemp. Phys. 42, 223 (2007). 